\title{
Prognostic and diagnostic value of procalcitonin in the post-transplant setting after liver transplantation
}

Aristotelis Perrakis, Falk Stirkat, Roland S. Croner, Nikolaos Vassos, Dimitrios Raptis, Süleyman Yedibela, Werner Hohenberger, Volker Müller

Department of Surgery, University Hospital Erlangen, Erlangen, Germany

Submitted: 19 September 2014

Accepted: 24 November 2014

Arch Med Sci 2016; 12, 2: 372-379

DOI: 10.5114 /aoms.2016.59264

Copyright @ 2016 Termedia \& Banach

\section{Abstract}

Introduction: The aim of the study was to assess the diagnostic accuracy of procalcitonin (PCT) as a marker for complications and as a prognostic factor for mortality after liver transplantation.

Material and methods: Liver transplant patients between January 2007 and April 2011 were prospectively included in the study. Procalcitonin serum concentration was recorded before, $6 \mathrm{~h}$ after reperfusion and then daily. Postoperative clinical course was prospectively analyzed from admission to discharge. Main surgical data such as operating procedure, type of reperfusion, operating and ischemic times, high urgency (HU) status and MELD score at the time of transplantation were also recorded.

Results: Sixteen patients with initial PCT $>5 \mathrm{ng} / \mathrm{ml}$ suffered $\geq 1$ complication $(p=0.03)$. However, there was no association between the level of the $1^{\text {st }}$ peak PCT and the further postoperative course or the occurrence of complications. Patients in whom a $2^{\text {nd }} \mathrm{PCT}$ peak occurred had a significantly higher risk for a complicated course, for a complicated sepsis course and for mortality $(p<0.0001)$. Warm ischemic time over $58 \mathrm{~min}$, operating time over $389 \mathrm{~min}$ and HU status were significant independent factors for a complicated postoperative course ( $p<0.001, p<0.001$ and $p=0.03$ respectively). Conclusions: Based on our results, we believe that PCT course and the occurrence of a $2^{\text {nd }}$ peak seem to possess important diagnostic and prognostic power in the post-transplant setting after liver transplantation.

Key words: procalcitonin, liver transplantation, infection, complication, sepsis.

\section{Introduction}

A systemic infection represents the most common complication in the early postoperative phase after orthotopic liver transplantation (LT) and induces an impaired post-transplant course associated with increased morbidity and mortality [1-7]. In the vast majority of cases, patients under immunosuppression did not show clinical signs of infection, such as fever, hypothermia, or tachycardia, and if these were present, they did not have any relevant specific diagnostic power, as they might indicate for example an impairment of the graft function such as an acute rejection episode [7]. Furthermore, well-established biochemical inflammation markers, such as C-reactive protein (CRP) or leucocyte count (LC), cannot immediately lead to the diagnosis of an infectious complication,

\author{
Corresponding author: \\ Aristotelis Perrakis MD \\ Department of Surgery \\ University of Erlangen- \\ Nuremberg \\ Krankenhausstr. 12 \\ 91054 Erlangen, Germany \\ Phone: +49 1723796295 \\ Fax: +49 91318536294 \\ E-mail: aristotelis.perrakis@ \\ uk-erlangen.de
}


whereby the early establishment of diagnosis is the most crucial stage in the management of such a patient population $[3,8]$.

Procalcitonin (PCT) is identified as a diagnostic marker for infectious or septic processes and correlates better with its severity than CRP [3, 8-13] Procalcitonin is an acute phase protein, composed of 116 amino acids, and is the precursor of calcitonin. In healthy individuals, PCT serum concentrations are very low $(<0.5 \mathrm{mg} / \mathrm{dl})$. The half-life of PCT is approximately 26 to $30 \mathrm{~h}[10,11,14]$. Neuroendocrine cells of solid organs (lung, kidney, pancreas, adrenal gland, and liver) might be the source of PCT during an inflammatory procedure. A further role might be played by extrathyroid cells, such macrophages, monocytes and liver cells in the PCT production during sepsis [14]. The main hypothesis concerning PCT induction proposes that the stimulation for PCT elevation is a result of a systemic challenge of the organism with bacterial endotoxin and bacterial polysaccharides [11]. Overall the exact pathophysiology is still an open matter [9-12]. In the post-transplant setting, elevated PCT levels are observed in the first 2-3 days after LT [7, 9-14]. Initially high PCT levels, however, do not necessarily indicate a poor prognosis as do continuous or secondarily rising levels [5-15]. Cardiac arrest and infection, but not PCT level in the donor, are associated with high post-LT PCT levels in the recipient [16]. In patients with impaired hepatic synthesis, PCT levels were not lower; they displayed the same predictive relevance for infection as in septic patients without cirrhosis or impairment of liver function [17]. On the other hand, PCT fails to predict an acute rejection episode [4, 7]. Furthermore an antithymocyte globulin (ATG)-based immunosuppressive therapy is a stimulus for synthesis and further elevation of PCT [18]. This fact represents the major limitation of PCT's diagnostic power. Additionally, the presence of viral infection did not stimulate a PCT elevation and cannot be diagnosed through this inflammation marker [1, 2, 7-10, 12, 13].

The aim of this study was to assess the prognostic value and accuracy of PCT as a marker for infectious and non-infectious postoperative complications, septic episodes and as a predictive factor for in hospital mortality in the immediate post-transplant setting in a cohort of deceased donor LT recipients. Furthermore, the impact of several aspects, such as ischemic, operating times, age, gender, Model of End stage Liver Disease (MELD) score, and high urgency (HU) status, which could induce a complication and/or a PCT elevation, have also been examined.

\section{Material and methods}

It was an observational study approved by the local ethics committee, since the measurement of PCT is a standard and well-established procedure in the intermediate care unit (ICU). Patients $(n=65)$ admitted for liver transplantation at our Surgical Department, Department of Hepatobiliary and Transplant Surgery between January 2007 and April 2011 were prospectively included in the study. Candidates for deceased donor LT were selected according to the national guidelines [19]. Patients with irreversible end-stage liver disease that was life-threatening and refractory to other forms of conventional medical or surgical therapy who had no contraindications for transplantation were regarded as eligible for LT. The organs were allocated according to the MELD [20].

For each recipient, the following were recorded: age, gender and PCT serum concentration before, $6 \mathrm{~h}$ after reperfusion and then daily during the stay in the ICU after LT. Postoperative clinical course was prospectively analyzed from admission to discharge according to the main clinical data: infectious/non-infectious complications, septic episodes, graft disorders, acute rejection episodes, renal failure, multiorgan failure and need for re-transplantation. Complications were graded according to the classification of Dindo et al. [21]. Furthermore, main surgical data such as operating procedure (piggyback technique, retrocaval resection technique), type of reperfusion (simultaneous $=$ porto-arterial/sequential $=$ portal), operating time, cold/warm ischemic time, HU status and MELD score at time of transplantation have been evaluated. The donor organ was always retrieved as part of a multiorgan donation. Either University of Wisconsin (UW) or histidine-tryptophan-ketoglutarate (HTK) solution was used for preservation.

\section{Procalcitonin measurement}

Blood samples were obtained for routine testing (biochemical parameters), and for each patient, serum aliquots were used for PCT determination. The procalcitonin levels were measured by an immunoluminometric LUMI test PCT kit (Brahms; Diagnostica, Berlin, Germany), which shows a detection range from 0.2 to $800.00 \mathrm{ng} / \mathrm{ml}$; the normal range detected was from 0.1 to $0.5 \mathrm{ng} / \mathrm{ml}$.

\section{Definitions}

Graft dysfunction was defined as the occurrence of at least one of the following criteria: the need for retransplantation (primary non-function - PNF), a rise in aminotransferases of above $2000 \mathrm{UI} / \mathrm{l}$, impairment of factor $\mathrm{V}(<30 \%)$ with synchronous increase of bilirubin without a retrospective need for retransplantation, serum bilirubin greater than $10 \mathrm{mg} / \mathrm{ml}$; PT of at least $17 \mathrm{~s}$; hepatic encephalopathy $[16,22]$. Mortality was defined as death from any cause occurring during the hospital stay. Pul- 
monary complication was defined as the need for mechanical ventilation. Acute renal failure was defined as plasma creatinemia of greater than $2 \mathrm{mg} /$ $\mathrm{dl}$ and urine output of less than $0.5 \mathrm{ml} / \mathrm{h}$. Renal complication was defined as the need for dialysis after LT or greater than $100 \%$ of creatinine levels compared with preoperative values. Postoperative complication was defined as hepatic dysfunction, infection, or pulmonary or renal complication or other surgical complication such as bleeding, hepatic artery thrombosis (HAT), thrombosis of the portal or cava vein and biliary complication such as insufficiency, stenosis, ischemic type biliary lesion (ITBL). The definition of clinical infection was standardized with criteria proposed by the Centers for Disease Control and Prevention and included pulmonary, bloodstream, or intra-abdominal infections accompanied by clinical symptoms proven by microbiological, radiological, or surgical findings and reacting to instituted therapy. Infection was diagnosed if clinical, biochemical or radiologic signs of infection were evident. Chest X-rays and ultrasound examinations were performed on a daily basis during the ICU stay and computed tomography (CT) scans when a clinical infection was suspected, and on the basis of these examinations, prompt therapy was initiated. In cases where samples from the suspected site of infection were positive, a proven infection could be defined. Sepsis was defined by the presence of infection and systemic inflammatory response syndrome (SIRS). The criteria for SIRS were met if more than one of the following signs occurred: body temperature greater than $38^{\circ} \mathrm{C}$ or less than $36^{\circ} \mathrm{C}$, heart rate greater than 100 beats per minute, hyperventilation as evidenced by a respiratory rate greater than 20 breaths per minute or a partial pressure of carbon dioxide in the arterial blood less than $32 \mathrm{~mm} \mathrm{Hg}$, and an LC greater than 10000 cells $/ \mu$ or less than 4000 cells $/ \mu l$. Severe sepsis was defined as sepsis complicated by organ dysfunction [23].

\section{Immunosuppression protocol, antibiotic prophylaxis}

All patients were initially treated with tacrolimus, starting $36 \mathrm{~h}$ after transplantation at $0.1 \mathrm{mg} / \mathrm{kg}$ twice daily and $500 \mathrm{mg}$ methylprednisolone in the anhepatic phase. All patients received basiliximab (Simulect, $20 \mathrm{mg}$ ) in the anhepatic phase followed by a second administration ( $20 \mathrm{mg}$ ) 4 days after transplantation. Mycophenolate mofetil $(500 \mathrm{mg}$ twice daily, intravenous or per oral) was administered starting on the $5^{\text {th }}$ postoperative day. Acute rejection was diagnosed based on the histopathological examination after liver biopsy according to BANFF criteria [24]. In 18 patients a conversion of the immunosuppressive regimen to ciclosporin-based immunosuppression was performed due to tacrolimus neurotoxicity. All recipients received broad-spectrum antimicrobial prophylaxis, consisting of antibacterial, antiviral, and antimycotic agents: with piperacillin-tazobactam for 7 days aciclovir and anidulafungin/posaconazole. Selective digestive decontamination consisted of $200 \mathrm{mg}$ of oral amphotericin B 3 times daily until the $21^{\text {st }}$ postoperative day. Furthermore, high-risk patients, recipients of a cytomegalovirus (CMV)-positive donor, received pre-emptive antiviral treatment with ganciclovir/valganciclovir adjusted to renal function over a 6-week course. The standard laboratory workup included hematologic and biochemical parameters. The CMV status (viral load, pp65 antigen) was examined twice a week. Cytomegalovirus (CMV) infection was defined by the appearance of CMV antigen polymerase chain reaction in the blood. X-ray examinations of the chest and ultrasounds of the graft were performed daily.

\section{Statistical analysis}

Values were expressed as median and range (minimum and maximum). Linear regression was used in univariate analysis to identify predictors of elevated PCT concentrations. Multivariate analysis was carried out using a logistic regression model. All of the tests performed were two-sided. Significant predictors in univariate analysis were included in a multivariate linear regression model, with a stepwise variable selection method. Potential associations between infectious complications or overall complications and surgical, clinical or biological parameters were tested with univariate procedures, using Students' paired t-test for continuous variables and Pearson's $\chi^{2}$ test for categorical variables. $P$-values of less than 0.05 were considered to be statistically significant. The first measured PCT value was defined as the beginning of observation. The end of the ICU stay or the occurrence of a second peak in the PCT level after LT was defined as the endpoint. A second peak was defined as an increase in PCT level after a previous decline from the first peak. The magnitude of increase was not defined. Postoperative mortality and morbidity were plotted against the occurrence of a second peak, the magnitude of the first peak, as well as the magnitude of the first PCT value measured. Statistical analysis was done with SPSS (SPSS v19.0, Chicago, IL, USA).

\section{Results}

Between January 2007 and April 2011, 65 patients with end-stage liver disease underwent 75 LT in the Surgical Department of the University of Erlangen-Nuremberg. The analyzed patient data were identified from a prospective database. Mean patient age was 53 years (range: 17-70). 
The sex bias was $64.6 \%: 35.4 \%(\operatorname{men}(n=42)$ : women $(n=23)$ (Table I).

\section{Surgical procedures, indication for liver transplantation}

All patients were indicated for LT because of end stage liver disease. Indications for LT were: 1. acute liver failure (ALF, $n=7,10.8 \%$ ), 2. hepatocellular carcinoma fulfilling the Milan criteria (HCC, $n=17,26.2 \%$ ), 3. hepatitis C induced liver cirrhosis (HCV, $n=13,20 \%)$, 4. hepatitis B induced liver cirrhosis (HBV, $n=3,4.8 \%$ ), 5. nutritive toxic liver cirrhosis (NTLC, $n=21,32 \%$ ), 6. hemochromatosis ( $n=2,3.1 \%), 7$. autoimmune hepatitis ( $n=2,3.1 \%$, Table I). The median MELD score was 31 (range: 12-40) and 21 LT were performed after acceptance of a request for $\mathrm{HU}$ liver transplantation (11 primary $\mathrm{HU}$ and $10 \mathrm{HU}$ after graft loss due to PNF or HAT, Table I). The donor organ was always retrieved as part of a multiorgan donation. Either University of Wisconsin ( $n=18)$ or histidine-tryptophan-ketoglutarate solution $(n=57)$ was used for preservation. Liver transplantation was performed by using either the piggyback technique $(n=30)$ or the retrohepatic caval resection tech- nique $(n=45)$. The median operating time was $320 \mathrm{~min}$ (range: 167-618 min). The median cold ischemic time was $560 \mathrm{~min}$ (range: 240-983 min), and the median warm ischemic time was $64 \mathrm{~min}$ (range: 29-158 min, Table I).

\section{Postoperative course}

Six acute rejection episodes were registered. Seven patients underwent 10 liver retransplantations because of primary graft non-function $(n=7)$ and hepatic artery thrombosis (HAT, $n=3$ ). Eighteen patients, who underwent 24 transplantations, died within 1 month during the postoperative course. At the end of the follow-up, 43 patients were alive, whereas 4 patients died during the follow-up and after discharge ( 2 because of cardiac arrest, 1 because of lung edema and 1 because of pneumonia). The median follow-up time was 16 months (range: 8-51 months). In 65 patients the peak PCT occurred within $72 \mathrm{~h}$ after LT. Initial PCT values $>5 \mathrm{ng} / \mathrm{ml}$ were observed among 24 patients with 26 LT, including 16 with 18 LT who suffered more than 1 complication (infectious, non-infectious). The odds ratio (OR) for a complicated course in the presence of an initial

Table I. Clinicopathological parameters of patients included in study

\begin{tabular}{|c|c|c|c|c|}
\hline Parameter & $\begin{array}{c}\text { All recipients } \\
n(\%)\end{array}$ & $\begin{array}{c}\text { No complications } \\
n(\%)\end{array}$ & $\begin{array}{l}\text { Recipients with } \\
\text { infectious } \\
\text { complications* } \\
n(\%)\end{array}$ & $\begin{array}{c}\text { Recipients with } \\
\text { non-infectious } \\
\text { complications } \\
n(\%)\end{array}$ \\
\hline Patients & $65(100 \%)$ & $34(52.3 \%)$ & $23(35.3 \%)$ & 19 (29.2\%) \\
\hline \multicolumn{5}{|l|}{ Gender: } \\
\hline Male & $42(64.6 \%)$ & $21(61.7 \%)$ & $15(65.2 \%)$ & $12(63.1 \%)$ \\
\hline Female & $23(35.4 \%)$ & $13(38.3 \%)$ & $8(34.8 \%)$ & 7 (36.9\%) \\
\hline Age [years] & $53(17-70)$ & $54(19-70)$ & $53(17-70)$ & $50(27-67)$ \\
\hline MELD (mean) & 31 & 26 & 34 & 32 \\
\hline HU (primary) & 11 & 4 & 7 & 7 \\
\hline \multicolumn{5}{|l|}{ Indication: } \\
\hline ALF & 7 (10.8\%) & $2(5.9 \%)$ & $4(17.4 \%)$ & $3(15.7 \%)$ \\
\hline $\mathrm{HCC}$ & $17(26.2 \%)$ & $11(32.3 \%)$ & $6(26.2 \%)$ & $3(15.7 \%)$ \\
\hline $\mathrm{VH}$ & $16(24.5 \%)$ & $10(29.5 \%)$ & $2(8.7 \%)$ & $4(21.2 \%)$ \\
\hline $\mathrm{AIC}$ & 7 (10.8\%) & $4(11.7 \%)$ & $5(21.7 \%)$ & $2(10.6 \%)$ \\
\hline Toxic & $14(21.5 \%)$ & $5(14.8 \%)$ & $5(21.7 \%)$ & $6(31.6 \%)$ \\
\hline HEM & $2(3.1 \%)$ & $1(2.9 \%)$ & $0(0 \%)$ & $1(5.2 \%)$ \\
\hline Others & $2(3.1 \%)$ & $1(2.9 \%)$ & $1(4.3 \%)$ & 0 \\
\hline CIT [min] & $560(240-983)$ & $552(240-972)$ & $568(247-983)$ & $572(267-945)$ \\
\hline WIT [min] & $64(29-158)$ & 47 (29-97) & $79(41-158)$ & $69(51-107)$ \\
\hline OT [min] & $320(167-618)$ & $245(167-418)$ & 389 (180-618) & 377 (170-593) \\
\hline
\end{tabular}

${ }^{\star}$ More complications in 1 patient possible. 
Table II. Association between $2^{\text {nd }}$ PCT peak and mortality

\begin{tabular}{|c|c|c|c|c|}
\hline Parameter & $\begin{array}{l}\text { All patients } \\
\text { with } 2^{\text {nd }} \text { peak } \\
\quad(n=25)\end{array}$ & $\begin{array}{l}\text { Alive group } \\
\quad\left(n=47^{*}\right)\end{array}$ & $\begin{array}{l}\text { Deceased group } \\
\qquad\left(n=18^{* *}\right)\end{array}$ & $P$-value \\
\hline $\begin{array}{l}\text { РCT level, mean } \\
{[\mathrm{ng} / \mathrm{ml}]}\end{array}$ & 45.41 & 24 & 61.98 & $<0.05$ \\
\hline РСТ level, range & $4.15-411.75$ & $4.15-54.41$ & $5.72-411.75$ & $<0.05$ \\
\hline $\begin{array}{l}\text { Highest PCT level } \\
{[\mathrm{ng} / \mathrm{ml}]}\end{array}$ & & 54.41 & 411.75 & $<0.05$ \\
\hline $\begin{array}{l}\text { Lowest PCT level } \\
{[\mathrm{ng} / \mathrm{ml}]}\end{array}$ & & 41 & 5.72 & 0.08 \\
\hline
\end{tabular}

${ }^{*}$ Concerns alive patients between $L T$ and discharge. ${ }^{* *}$ Concerns in-hospital mortality.

Table III. Postoperative (infectious and non-infectious) complications according to Clavien-Dindo classification after LT

\begin{tabular}{|lc|}
\hline Grade of complication & N \\
\hline I & 4 \\
\hline II & 5 \\
\hline IIIla & 9 \\
\hline IVa & 3 \\
\hline IVb & 3 \\
\hline V & 4 \\
\hline Total & 14 \\
\hline
\end{tabular}

PCT > $5 \mathrm{ng} / \mathrm{ml}$ was calculated to be $62.5 \%(95 \%$ confidence interval, $11.3-12.1 ; p=0.03)$. Among these patients with initial PCT values $>5 \mathrm{ng} / \mathrm{ml}$, 8 patients displayed uneventful postoperative courses. Thirty-one patients suffered $42 \mathrm{com}$ plications (infectious, non-infectious) overall, and there was a significant association with the occurrence of a $2^{\text {nd }} P C T$ peak $\left(O R=64.60 \%, \chi^{2}\right.$ Pearson $=15.08, p<0.001)$. In patients with a graft dysfunction, including acute rejection episodes $(n=16)$, there was a significant PCT elevation with the occurrence of a $2^{\text {nd }}$ peak $(O R=$ $24.6 \%, \chi^{2}$ Pearson $\left.=16.416, p<0.0001\right)$. However, in 10 patients there was a coincidence of an infectious focus. Among 7 patients with a PNF and the need of retransplantation, in 4 patients there was a significant elevation of PCT with the occurrence of a $2^{\text {nd }}$ peak.

In contrast, in patients with a HAT a $2^{\text {nd }}$ PCT peak was not registered. Patients with bile duct complications (ITBL, stenosis, insufficiency) did not show elevation of PCT or a $2^{\text {nd }}$ peak (OR $=13.9 \%$, $\chi^{2}$ Pearson $=0.428, p=0.388$ ), although there was an elevation of the liver enzymes. Furthermore, there was no overall correlation between PCT and parameters of liver function, whereas other immunological parameters, such as antibodies in the case of autoimmune hepatitis, had no correlation with the PCT course.

Twenty-three patients experienced acute renal failure after LT with the intermittent need for continuous veno-venous hemofiltration (CVVH), but there was no association between this complication and the PCT course. Furthermore, the risk of renal failure requiring hemofiltration was independent of a PCT > $5 \mathrm{ng} / \mathrm{ml}(\mathrm{OR}=8.25 ; p<0.04$; $\chi^{2}$ Pearson $\left.=4.31\right)$. There was no association between the $1^{\text {st }}$ peak PCT and the further postoperative course or the occurrence of complications (infectious or non-infectious, $p=0.442$ ). Patients in whom a $2^{\text {nd }}$ PCT peak occurred had a significantly higher risk for a complicated course $(p=0.01)$, for a complicated sepsis course with multiorgan dysfunction $\left(n=22, \mathrm{OR}=33.90 \%, \chi^{2}\right.$ Pearson $=$ 34.016, $p<0.0001$ ), for a non-infectious complication $(p<0.0001)$ and for in-hospital mortality $(p<0.0001$, Tables II and III). Comparing the different surgical techniques - piggyback technique $(n=30)$ or retrohepatic caval resection technique $(n=45)$, the use of different preservation solution (HTK vs. UW) and the reperfusion types (portal vs. porto-arterial), we did not observe any significant difference in the occurrence of complications or the PCT course (infectious, non-infectious, OR: $6.9 \%, \chi^{2}=0.328, p=0.638 ; \mathrm{OR}=7.4 \%, \chi^{2}=0.461$, $p=0.759 ;$ and $\mathrm{OR}=8.9 \%, \chi^{2}=0.625, p=0.529$ respectively, Table IV). Warm ischemic time over 58 min, operating time over 389 min and HU status were significant independent factors for a complicated postoperative course (infectious and non-infectious complications, $p<0.001, p<0.001$ and $p=0.03$ respectively). In contrast, cold ischemic time, age, MELD score, and indication for LT were not significant factors for postoperative complications or the course of PCT ( $p=0.289, p=0.479$, $p=0.08$ and 0.118 respectively).

\section{Discussion}

In the MELD era and due to the shortage of available organs, which has led to the need to accept marginal organs, the risk factors which could 
Table IV. Operating procedures and their influence on outcome

\begin{tabular}{|lcccc|}
\hline Procedure & All LT $(n=75)$ & $\begin{array}{c}\text { Infectious } \\
\text { complications }\end{array}$ & $\begin{array}{c}\text { Non-infectious } \\
\text { complications }\end{array}$ & $P$-value \\
\hline \begin{tabular}{l} 
Operating technique: \\
\hline Cava resection
\end{tabular} & 45 & 14 & 10 & 0.137 \\
\hline Piggy back & 30 & 9 & 8 & 0.167 \\
\hline \begin{tabular}{l} 
Type of reperfusion: \\
\hline Simultaneous
\end{tabular} & 44 & 16 & 9 & 0.362 \\
\hline Portal & 31 & 8 & 6 & 0.274 \\
\hline \begin{tabular}{l} 
Preservation solution: \\
\hline HTK
\end{tabular} & 57 & & 12 & 0.596 \\
\hline UW & 18 & 9 & 2 & 0.471 \\
\hline
\end{tabular}

lead to an impaired post-transplant setting are significantly more numerous. Therefore accurate diagnosis of postoperative complications and immediate initiation of treatment are crucial for the further outcome $[5,15,16,20]$. Procalcitonin is a well-established diagnostic marker for surgical patients, and its levels correlate with the severity of complications and SIRS or sepsis [8]. However, there has been no systemic use of its diagnostic power in the postoperative management of patients in the post-transplant setting. Our results show that $L T$ recipients with infectious and some non-infectious complications had significantly higher values of PCT, in the majority of cases with a $2^{\text {nd }}$ peak value after the initial PCT peak, in comparison to recipients with an uncomplicated post-transplant course. The $1^{\text {st }}$ peak PCT was not an independent risk factor in LT recipients, while the occurrence of a $2^{\text {nd }}$ peak was a significant independent factor for protracted septic course, for any complication overall (infectious or non-infectious) and for post-transplant mortality (Table II). The fact that the $1^{\text {st }}$ PCT peak does not play a diagnostic and prognostic role was also underlined by Eyraud et al. [16]. In this study it was found that PCT level in the donor and early PCT peak in the recipient do not have predictive value for post-LT hepatic dysfunction or other complications. Cardiac arrest and infection in the donor seem to be associated with high post-LT PCT levels in the recipient. Another limitation in the diagnostic accuracy of PCT was noted by Zazula et al., who reported the ATG-stimulated elevation of PCT [18]. We were not able to examine this aspect, as we prefer ATG-free immunosuppressive regimens. The occurrence of a $2^{\text {nd }}$ PCT peak and of an eventful post-transplant setting were significant in patients with severe sepsis, bacterial or fungal infection and graft dysfunction overall (PNF and acute rejection (AR)). In $A R$ alone there was not a prognostic value of PCT to register. Furthermore, warm ischemic and longer operating time were independent factors for eventful postoperative course with infectious and/or non-infectious complications and for occurrence of a $2^{\text {nd }}$ PCT peak. This fact explains why warm ischemia and reperfusion lead to a significant proinflammatory response resulting in tissue damage $[25,26]$. This underlines the importance of minimizing the operating times during LT. The first peak value of PCT, usually occurring on the $2^{\text {nd }}$ or $3^{\text {rd }}$ postoperative day, was not an independent factor for a fatal outcome according to the present series, and this finding confirms our earlier results [15]. An initially high PCT has been described not to indicate a poor prognosis when followed by an adequate decline $[5,7,15,16]$. A rapidly rising PCT without a decline after the $3^{\text {rd }}$ postoperative day was associated with a fatal outcome correlating with a bacterial or fungal infection [7, 15]. This fact was also registered in the present series [7, 15]. In contrast to earlier studies where an elevation of PCT was seen only in infectious complications [5, 7], we also observed a greater number of non-infectious complications when the PCT was elevated or showed a $2^{\text {nd }}$ peak. Our study included an acceptable cohort of deceased donor LT recipients and showed that PCT was an independent risk factor for infectious and some non-infectious complications and that the occurrence of a $2^{\text {nd }}$ peak was an independent factor for a complicated postoperative outcome and for mortality (Table II). Furthermore, we registered a high prognostic value, due to its occurrence before clinical onset of the complications and clinical impairment of the patients. Various studies have demonstrated that PCT is a useful diagnostic marker for bacterial and fungal infections and sepsis in different patient groups, with superior diagnostic accuracy in comparison with CRP $[7,17,27,28]$. Furthermore, PCT was described in some small cohort studies as an accurate marker for any complication in the post-transplant setting [27, 29, 30], which could not be fully confirmed by other study groups or by the present series [5, 15-17]. Van den Broek et al. in a large study combining a large cohort of living and deceased donor LT recipients 
showed that although median peak PCT levels differed significantly between LT recipients with and without clinically significant infections, peak PCT was not an independent risk factor for serious infections in the early post-transplant period, had an inferior diagnostic accuracy compared to other inflammation markers, such as CRP, and increased the prognostic accuracy only marginally. Furthermore, they stated that no second peak in the PCT level could be observed in the majority of recipients with a critical infection before its onset. We could not confirm their results, as we found that PCT was a highly significant, independent factor with very high prognostic and diagnostic accuracy. In the same study 5 independent factors for infectious complications were underlined [5]. We confirmed that the ischemic times play an important role for the further postoperative outcome. The different results might be a result of the many living donor LT recipients and the completely different pathophysiological processes in comparison to the deceased donor recipients. One limitation of the present study was the fact that only deceased donor LT recipients were evaluated. Another limitation is that this study was performed in a single institution and the results obtained might not be comparable to those in other centers with a larger population such as that of Van de Broek et al. Single-center studies, however, have the advantage of reducing the number of possible differences in surgical technique and postoperative management procedures, and we believe that our cohort included an acceptable number of patients in order to have a reliable statistical analysis. Several reports have underlined the fact that the initial PCT values and the peak PCT are dependent on the infection status and the cardiac arrest of the donor [16]. We could confirm this thesis. However, this study reports the significance of the role of PCT as an independent factor as regards its course and the $2^{\text {nd }}$ peak. Nevertheless, the data reported included all patients of our Transplant Center with no exclusions and underline the prognostic and diagnostic importance of PCT. We have to admit that PCT must not be regarded as the only reliable diagnostic parameter. Postoperative complications after liver transplantation may result from multifactorial processes, which cannot be monitored by only 1 marker. Based on our results, we believe that the value of PCT and of the occurrence of a $2^{\text {nd }}$ peak seem to be important diagnostic tools for the early detection of serious infectious complications. This fact is of great importance in the post-transplant management of a patient under immunosuppression, with a high risk of serious outcomes, such as a fatal septic shock. Therefore, the immediate diagnosis and the proper therapeutic reaction are the keys to prevent mortality and a complicated postoperative course. The correlation between PCT course, clinical course, other markers such as CRP and other diagnostic tools must be evaluated to ensure detection of early signs of a postoperative complication.

\section{Acknowledgments}

Above all, I would like to thank my teacher and supervisor Dr. Giovanni-Günther Braun (1948-2011), head of the Intensive Care Unit of the Surgical Department of the University of Erlangen-Nuremberg, who taught me with patience the principles of sepsis, contributed enormously to my development and showed me how to gain the clinical view and to offer the optimal care in patients with severe sepsis.

I would like to especially thank Elli Perraki, Statistics and Operational Research, University of Essex UK, for her essential contribution to the statistical analysis.

Perrakis $A$ and Stirkat $F$ contributed equally to this work.

\section{Conflict of interest}

The authors declare no conflict of interest.

\section{References}

1. lida T, Kaido T, Yagi S, et al. Posttransplantbacteremia in adult living donor liver transplant recipients. Liver Transpl 2010; 16: 1379-85.

2. Lee SO, Kang SH, Abdel-Massih RC, Brown RA, Razonable RR. Spectrum of early onset and late-onset bacteremias after liver transplantation: implications for management. Liver Transpl 2011; 17: 733-41.

3. Castelli GP, Pognani C, Meisner M, Stuani A, Bellomi D, Sgarbi L. Procalcitonin and C-reactive protein during systemic inflammatory response syndrome, sepsis and organ dysfunction. Crit Care 2004; 8: 234-42.

4. Kornberg A, Grube T, Wagner T, et al. Differentiated therapy with prostaglandin E1 (alprostadil) after orthotopic liver transplantation: the usefulness of procalcitonin (PCT) and hepatic artery resistive index (RI) for the evaluation of early graft function and clinical course. Clin Chem Lab Med 2000; 38: 1177-80.

5. Van den Broek MA, Olde Damnik SW, Winkens B, et al. Procalcitonin as a prognostic marker for infectious complications in liver transplant recipients in an Intensive Care Unit. Liver Transpl 2010; 16: 402-10.

6. Saner FH, Olde Damink SW, Pavlakovic G, et al. Pulmonary and blood stream infections in adult living donorand cadaveric liver transplant patients. Transplantation 2008; 85:1564-8.

7. Kuse ER, Langefeld I, Jaeger K, Kulpmann WR. Procalcitonin in fever of unknown origin after liver transplantation: a variable to differentiate acute rejection from infection. Crit Care Med 2000; 28: 555-9.

8. Meisner M, Tschaikowsky K, Hutzler A, Schick C, Schuttler J. Postoperative plasma concentrations of procalcitonin after different types of surgery. Intensive Care Med 1998; 24: 680-4. 
9. Morgenthaler NG, Struck J, Chancerelle Y, et al. Production of procalcitonin (PCT) in non-thyroidal tissue after LPS injection. Horm Metab Res 2003; 35: 290-5.

10. Meisner M, Tschaikowsky K, Schmidt J, Schüttler J. Procalcitonin (PCT)-indications for a new diagnostic parameter of severe bacterial infection and sepsis intransplantation, immunosuppression and cardiac assist devices. Cardiovascular Engineering 1996; 1: 67-76.

11. Assicot M, Gendrel D, Carsin H, Raymond J, Guilbaud J, Bohuon C. High serum procalcitonin concentrations in patients with sepsis and infection. Lancet 1993; 341: 515-8.

12. Meisner M, Tschaikowsky K, Palmaers T, Schmidt J. Comparison of procalcitonin (PCT) and C-reactive protein (CRP) plasma concentrations at different SOFA scores during the course of sepsis and MODS. Crit Care 1999; 3: 45-50.

13. Arkader R, Troster EJ, Lopes MR, et al. Procalcitonin does discriminate between sepsis and systemic inflammatory response syndrome. Arch Dis Child 2006; 91: 117-20.

14. Brunkhorst FM, Heinz U, Forycki ZF. Kinetics of procalcitonin in iatrogenic sepsis. Intensive Care Med 1998; 24: 888-9.

15. Perrakis A, Yedibela S, Schellerer V, Hohenberger W, Müller V. Procalcitonin in the setting of complicated postoperative course after liver transplantation. Transplant Proc 2010; 42: 4187-90.

16. Eyraud D, Ben Ayed S, Tanguy ML, et al. Procalcitonin in liver transplantation: are high levels due to donors or recipients? Crit Care 2008; 12: R85.

17. Bota DP, Van Nuffelen M, Zakariah AN, Vincent JL. Serum levels of C-reactive protein and procalcitonin in critically ill patients with cirrhosis of the liver. J Lab Clin Med 2005; 146: 347-51.

18. Zazula R, Prucha M, Tyll T, Kieslichova E. Induction of procalcitonin in liver transplant patients treated with anti-thymocyte globulin. Crit Care 2007; 11: R131.

19. Bundesärztekammer, Richtlinien zur Organtransplantation § 16 (Allgemeiner teillebertransplantation http:// baek.de/downloads/riliorgaleber20110326).

20. Wiesner R, Edwards E, Freeman R, et al. United Network for Organ Sharing Liver Disease Severity Score Committee Model for end stage liver disease (MELD) and allocation of donor livers. Gastroenterology 2003; 124: 91-6.

21. Dindo D, Demartines N, Clavien PA. Classification of surgical complications: a new proposal with evaluation in a cohort of 6336 patients and results of a survey. Ann Surg 2004; 240: 205-13.

22. Deschênes M, Belle SH, Krom RA, Zetterman RK, Lake JR. Early allograft dysfunction after liver transplantation: a definition and predictors of outcome. National Institute of Diabetes and Digestive and Kidney Diseases Liver Transplantation Database. Transplantation 1998; 66: 302-10

23. Calandra T, Cohen J. International Sepsis Forum Definition of Infection in the ICU consensus Conference The international sepsis forum consensus conference on definitions of infection in the intensive care unit. Crit Care Med 2005; 33: 1538-48.

24. BANFF schema for grading liver allograft rejection: an international consensus document. Hepatology 1997; 25: 658-63.

25. Dogan S, Aslan M. Hepatic ischemia-reperfusion injury and therapeutic strategies to alleviate cellular damage. Hepatol Res 2011; 41: 103-17.

26. Jaeschke H. Molecular mechanisms of hepatic ischemia reperfusion injury and preconditioning. Am J Physiol Gastrointest Liver Physiol 2003; 284: G15-26.
27. Simon L, Gauvin F, Amre DK, Saint-Louis P, Lacroix J. Serum procalcitonin and C-reactive protein levels as markers of bacterial infection: a systematic review and metaanalysis. Clin Infect Dis 2004; 39: 206-17.

28. Meisner M, Müller V, Khakpour Z, Toegel E, Redl H. Induction of procalcitonin and proinflammatory cytokines in an an hepatic baboon endotoxin shock model. Shock 2003; 19: 187-90.

29. Cooper D, Sharples L, Cornelissen J, Wallwork J, Alexander G, Trull A. Comparison between procalcitonin, serum amyloid $\mathrm{A}$, and C-reactive protein as markers of serious bacterial and fungal infections after solid organ transplantation. Transplant Proc 2001; 33: 1808-10.

30. Kunz D, Pross M, Konig W, Lippert H, Manger T. Diagnostic relevance of procalcitonin, IL-6 and cellular immune status in the early phase after liver transplantation. Transplant Proc 1998; 30: 2398-9. 